\title{
"Every day is just kind of weighing my options." Perspectives of young adult cancer survivors dealing with the uncertainty of the COVID-19 global pandemic
}

\author{
L. Aubree Shay ${ }^{1}$ (D) Marlyn Allicock ${ }^{2} \cdot{\text { Amanda } \mathrm{Li}^{1}}^{1}$ \\ Received: 2 March 2021 / Accepted: 1 June 2021 / Published online: 14 June 2021 \\ (C) The Author(s), under exclusive licence to Springer Science+Business Media, LLC, part of Springer Nature 2021
}

\begin{abstract}
Purpose We explored adolescent and young adult cancer survivor (AYA) experiences with COVID-19 to understand the impact of living through a pandemic, unmet needs, and coping strategies.

Methods AYAs were recruited nationally, completed an online survey, and attended one of six online focus groups. We used qualitative content analysis to analyze focus group data.

Results Thirty-nine AYAs completed the survey, and 24 also participated in the focus groups. In the survey, AYAs responded that COVID-19 increased anxiety about their health or their family's health, feelings of isolation, and worries about job security. Overarching focus group themes included AYA behavioral responses to the pandemic similar to their peers, the added burden of cancer, and unexpected advantages of a cancer history. When discussing the added burden of cancer, subthemes included difficulties and delays in medical care, mental health stressors, and compounding uncertainty. Unexpected advantages of a cancer history included relying on coping strategies developed during active treatment and resiliency from practicing social distancing during treatment.

Conclusions AYAs have struggled in the early pandemic in ways similar to their peers but with compounding uncertainty regarding their unknown risk due to cancer history. Healthcare providers and systems can better support AYAs by providing additional psychosocial supports, developing strategies to triage good candidates for telehealth, and providing information about cancer survivor-specific risks for COVID-19.

Implications for Cancer Survivors Our findings indicate a need for psychosocial supports that address managing anxiety and uncertainty. AYAs may be able to draw on their cancer experiences to navigate the COVID-19 pandemic.
\end{abstract}

Keywords Adolescent and young adult (AYA) $\cdot$ COVID-19 $\cdot$ Social distancing $\cdot$ Focus group $\cdot$ Qualitative

\section{Introduction}

Coronavirus (COVID-19) was first identified in Wuhan, China, in December 2019 [1]. Due to the rapid spread of the virus, the World Health Organization declared COVID-19 as a pandemic approximately 3 months later, on March 11, 2020 [2]. Individuals infected with COVID-19 may develop numerous symptoms ranging from mild to severe. However,

L. Aubree Shay

Laura.aubree.shay@uth.tmc.edu

1 UTHealth School of Public Health in San Antonio, 7411 John Smith Dr., Ste 1100, San Antonio, TX 78229, USA

2 UTHealth School of Public Health in Dallas, 5323 Harry Hines Blvd., V8.112, Dallas, TX 75390, USA symptoms most commonly associated with COVID-19 include shortness of breath, cough, fatigue, and fever or chills [3]. Male sex, older adults, individuals with comorbidities, and individuals diagnosed with cancer (regardless of treatment and cancer status) are at an increased risk of mortality and developing complications from COVID-19 [3, 4].

Cancer patients and survivors may have higher risks of contracting COVID-19 and developing adverse outcomes due to their compromised immune system caused by their cancer treatment [5]. Adolescent and young adult cancer survivors (AYAs) ranging from ages 15 to 39 represent a unique subset of the cancer population. Before the pandemic, studies had already highlighted the need for more survivorship programs explicitly catered to AYA cancer survivors [6]. Compared to adult and child cancer survivors, AYAs are historically an understudied and underserved group with unmet survivorship care needs $[6,7]$. With disruptions to healthcare 
settings and clinic flow, COVID-19 has exacerbated the healthcare needs of AYA cancer survivors as some cancer patients and survivors have experienced delayed treatment, canceled follow-up care, or changes to clinic routines [8]. Due to this increased risk and disruption to care, AYA patients and survivors may also experience increased stress and anxiety as they navigate social distancing and quarantine guidelines. Thus, to continue to improve care to AYAs, we need to develop a better understanding of the needs and experiences of AYA cancer survivors during the COVID-19 pandemic that may offer additional insight into coping and illness management.

Using a mixed methods approach that included a brief survey and focus groups with AYA cancer patients and survivors, this study aimed to explore AYA experiences with COVID-19 in the early stages of the pandemic, highlighting the patient voice in this turbulent time, to better understand the impact of living through a pandemic, unmet needs, and coping strategies.

\section{Methods}

\section{Procedure}

We recruited AYA cancer survivors via the email listserv and flyers posted by our community partner, Elephants and Tea, a media company focused on AYA cancer patients, survivors, and caregivers (https://elephantsandtea.com). Following the National Cancer Institute's definition of a cancer survivor, we included all AYAs, aged 18 to 39, with a history of a cancer diagnosis, regardless of time from diagnosis or treatment status. Because we were conducting an online focus group study, we chose to focus only on legal adults and exclude younger AYAs (aged 15-17 years) as they may not have been making their own healthcare and social distancing decisions. The recruitment information included a link for participants to indicate preferred times for the online focus group and complete a brief survey via REDCap. The link allowed for participants to provide consent before starting the survey. Survey items queried participants about demographic information (age, race/ethnicity, gender, marital status, education level, household income, employment status, current state of residence), perceived health status, and the impact of COVID-19 using a previously published 12-item measure $[9,10]$.

AYAs received an email confirming the receipt of their survey and an invitation to participate in a 90-min focus group led by two of the authors (LAS and MA). During June and July 2020, we contacted all AYAs who completed surveys, inviting them to join the focus group. Six AYA focus groups were conducted and recorded via WebEx and were professionally transcribed. Any participant notes written in the chat were later added to the transcripts with time stamps. All focus groups followed the same protocol. Eligibility criteria included self-reported cancer diagnosis and English speaking. Participants received a $\$ 40$ gift card for completing the survey and focus group. All study procedures were approved by the institutional review board at the University of Texas Health Science Center in Houston (HSC-SPH-20-0463).

\section{Instrument}

All focus groups followed the same semi-structured focus group guide (see Supplementary Materials). We developed the guide to assess the impact of COVID-19 in the early stages of the pandemic, how AYAs responded, issues related to cancer care and access, and barriers and coping strategies and resources during the ongoing pandemic.

\section{Data analysis}

Quantitative analysis Descriptive statistics were used to summarize sample characteristics and proportions impacted by specific factors related to COVID-19.

Qualitative analysis We used a thematic analysis to examine focus group data $[11,12]$. We used a qualitative content analysis where themes and categories are obtained from the data on the context of the pre-determined questions. Transcripts were independently reviewed and coded by all three authors and an additional research assistant. After coding, we worked together to find consensus on the themes.

\section{Results}

\section{Study participants}

In total, 39 AYAs with a history of cancer completed the survey. Of these, 24 participants also attended one of the six focus groups (Table 1). The focus groups lasted an average of $57 \mathrm{~min}$ (range $=25-92 \mathrm{~min}$ ). Comparing the groups of participants who completed the survey only and those that completed both the survey and attended a focus group, there was no significant difference $(p>.05)$ in gender, race/ethnicity, marital status, educational status, and annual household income. Both groups consisted of primarily female, White, collegeeducated participants with an average annual income of under $\$ 50,000$. AYAs who participated in both the survey and focus group were more likely to be students than those who completed the survey only $(p \leq .05)$.

In the online survey, we asked AYAs to indicate areas in which they have been impacted by COVID-19 (Table 2). The most commonly endorsed response options included "anxiety 
Table 1 Demographics of focus group attendees $(n=24)$ versus those who completed the survey but did not attend a focus group $(\mathrm{n}=15)$

Table 2 Survey responses on the impact of COVID-19

\begin{tabular}{|c|c|c|c|}
\hline & $\begin{array}{l}\text { Attended focus group } \\
(\mathrm{n}=24)\end{array}$ & $\begin{array}{l}\text { Survey only } \\
(\mathrm{n}=15)\end{array}$ & $\begin{array}{l}\text { Chi-square p- } \\
\text { value }\end{array}$ \\
\hline & & $\mathrm{n}(\%)$ & \\
\hline Female & $19(79 \%)$ & $11(73 \%)$ & $\mathrm{p}=0.67$ \\
\hline Race/ethnicity & & & $\mathrm{p}=0.67$ \\
\hline Asian & $2(8 \%)$ & $1(7 \%)$ & \\
\hline Black or African American & $0(--)$ & $1(7 \%)$ & \\
\hline Hispanic & $4(17 \%)$ & $3(20 \%)$ & \\
\hline $\begin{array}{l}\text { Native Hawaiian or Pacific } \\
\text { Islander }\end{array}$ & $1(4 \%)$ & $0(--)$ & \\
\hline White & $17(71 \%)$ & $10(67 \%)$ & \\
\hline Marital status & & & $\mathrm{p}=0.22$ \\
\hline Married or living with partner & $7(29 \%)$ & $10(67 \%)$ & \\
\hline Single & $17(71 \%)$ & $5(33 \%)$ & \\
\hline Educational status & & & $\mathrm{p}=0.90$ \\
\hline Some college or less & $8(33 \%)$ & $6(40 \%)$ & \\
\hline College graduate & $10(42 \%)$ & $6(40 \%)$ & \\
\hline Some graduate school or more & $6(25 \%)$ & $3(20 \%)$ & \\
\hline Annual household income & & & $\mathrm{p}=0.98$ \\
\hline Under $\$ 50,000$ & $9(38 \%)$ & $6(40 \%)$ & \\
\hline$\$ 50,000-\$ 99,999$ & $8(33 \%)$ & $5(33 \%)$ & \\
\hline$\$ 100,000$ or more & $7(29 \%)$ & $4(27 \%)$ & \\
\hline Occupational status & & & $\mathrm{p}=0.02$ \\
\hline Employed & $13(54 \%)$ & $12(80 \%)$ & \\
\hline Out of work/homemaker & $1(4 \%)$ & $3(20 \%)$ & \\
\hline Student & $7(29 \%)$ & $0(--)$ & \\
\hline Unable to work & $3(13 \%)$ & $0(--)$ & \\
\hline
\end{tabular}

\begin{tabular}{lc}
\hline & $\begin{array}{c}\text { Total (n=39) } \\
\mathrm{n}(\%)\end{array}$ \\
\hline Anxiety due to worry about my health or my family's health & $35(90 \%)$ \\
I feel more isolated & $25(64 \%)$ \\
Worried about job security & $18(46 \%)$ \\
I am less physically active & $15(39 \%)$ \\
Scheduling delays related to treatment & $14(36 \%)$ \\
Less sleep & $14(36 \%)$ \\
Decreased productivity at work & $14(36 \%)$ \\
Reduction in job hours or wages & $11(28 \%)$ \\
Reduced access to treatment services & $11(28 \%)$ \\
Delayed or reduced communication with my healthcare team & $11(28 \%)$ \\
Sleeping more & $8(21 \%)$ \\
Temporary laid off from work & $7(18 \%)$ \\
I have had trouble getting my medications & $6(15 \%)$ \\
Forced to use vacation or sick time & $3(8 \%)$ \\
I have had trouble getting food and other personal necessities & $3(7 \%)$ \\
Loss of employment & $2(5 \%)$ \\
\hline
\end{tabular}


due to worry about my health or my family's health" (90\%), "I feel more isolated" (64\%), and "worried about job security" $(46 \%)$.

\section{Focus group results}

Below we present our focus group results in four broad themes: AYA behavioral responses to the pandemic, the added burden due to cancer, the unexpected advantages of a cancer history, and AYA recommendations and resources (Table 3).

\section{AYA behavioral responses to pandemic}

When asked how they had changed their behavior response to the pandemic as of summer 2020, a commonly echoed response across most AYAs was adherence to social distancing and mask-wearing guidelines. For example, one AYA mentioned he started wearing masks "not when it became mandated but like when it became recommended by health officials" (Male, Focus Group (FG) 1). AYAs avoided unnecessary trips outside their homes and would only venture outside for required trips such as medical appointments and grocery shopping. However, if AYAs lived near family and friends, survivors had less susceptible individuals (e.g., partner, roommate) complete "outdoor" tasks. Additionally, if they were able to afford it, AYAs opted for curbside grocery pick-up or grocery delivery services over entering the store. Although they were concerned about their health, AYAs were also concerned for the health of others, such as their parents. For example, one stated, "I try to go to the grocery store for [my parents] because they're older, and I feel like I'm in better health than they are." (Male, FG 3).

If venturing outside in the early stages of the pandemic, AYAs feared bumping into anyone not wearing a mask and were "very serious about staying far away from people who aren't wearing face masks." (Female, FG 6). So much so that they felt like they had to be the "bad guy" in the family. For example, one said, "I'm definitely seen as the bad guy in my family, because I don't agree to birthday parties, I won't agree to taking my mask off, I won't go into people's houses, I won't let my child go into people's houses, I won't let people visit." (Female, FG 2). As a result, having laws and restrictions to limit social interaction and mandate mask-wearing helped reduce stress and burden on immunocompromised individuals.

Some AYAs considered themselves to be at a higher risk for contacting COVID-19 than the general public, due to being immunocompromised from cancer or cancer treatment or their cancer coupled with another underlying condition such as asthma. Because they perceived themselves to be at higher risk, this meant being hypervigilant with safety measures such as cleaning, determining where was safe to go, staying away from people without masks, and feeling "more paranoid" when going out. For others, it meant having to cut people off. For example, one AYA stated: "I'm still in treatment. I still have cancer. Like, they just wouldn't get it. And so, I had to cut some of those friends off, which was unfortunate." (Female, FG 3).

When AYAs were asked about the impacts of social distancing, they mentioned feeling frustration at others for not following mask wearing and social distancing guidelines, fear of contracting the virus, fear of infecting their loved ones, changes and disruptions in family dynamics, and changes in daily "normal" activities such as going for a swim. They also faced an internal struggle of what was the right thing to do. For example, one AYA said,

I had a really good friend that got married in May, and so I kind of had this internal conflict back and forth of, the wedding still happened, and I wanted to go, and so it was just...there's been a lot of situations and social situations like that that I feel like I've been having a lot of internal battle with what is the right thing to do. (Female, FG 4)

With the transition to remote work, remote learning, and smaller social circles, AYAs faced feelings of isolation, and some struggled with depression. Other negative impacts during the pandemic included lost job opportunities, changes in
Table 3 Focus group themes and subthemes

\begin{tabular}{ll}
\hline Themes & Subthemes \\
\hline Response to pandemic & Social distancing \\
& Impact of social distancing on AYAs \\
Added burden due to cancer & Difficulties and delays in medical care \\
& Mental health stressors \\
& Compounding uncertainty regarding an unknown timeline, \\
& risk perceptions, and weighing medical decisions \\
Unexpected advantages of a cancer history & Coping strategies \\
& Resiliency \\
AYA's recommendations and resources & \\
\hline
\end{tabular}


work roles, reduced work hours, and reduced incomes. For example, one AYA said, "So yeah, there has been job changes. And not only, like, my role changing, but hours lost, financial status, conversations just get weird." (Female, FG 3).

A bright spot for AYAs is they felt like those with cancer and those without cancer were now all in the same boat. Orders and policies helped to create peace and a perceived group mentality of we are all in this together. For example, one survivor said,

I was really glad when the order came down that all these places you have to wear a mask every time you go in there. Because I just felt a sense of kind of like peace, that even though there are people who fight that, that it's a we're all in it together type of thing. (Female, FG 2)

Other positive impacts/experiences during the pandemic included having more time to reconnect and develop closer relationships with family and friends, having more support from family and friends, and, most notably, connecting with friends and support groups that would not have been possible without the pandemic. With the cancellation of in-person resources survivors typically have access to, the circumstances surrounding the pandemic helped encourage AYAs to find new support outlets. Additionally, the transition to remote events and virtual conferences helped reduce barriers that often prevented survivors from attending in the first place. For example, one participant said,

So the cool thing, and I can't believe I'm actually saying it's a cool thing, about COVID is that Stupid Cancer had their CancerCon virtually this year. And it's something I probably would not have gone to in person, but I went to the digital CancerCon, and that's how I met [name redacted] And now I'm in an Instagram group chat with like a dozen other adolescent and young adult cancer survivors. I have my tribe. This is not something I had before this. You know, I'm almost four years out. Had not met a group of people, despite trying different things. This, it just wouldn't have happened without COVID and without having done the virtual conference." (Female, FG 3)

\section{Added burden due to cancer}

While AYAs experienced the early pandemic similar to their peers in many ways, they also described an added burden due to their history of cancer. These added burdens took the form of difficulties and delays in medical care, mental health stressors, and compounding uncertainty.
Difficulties and delays in medical care AYAs described several difficulties related to their medical care. Early in the pandemic, many oncology clinics attempted to reduce foot traffic by issuing new guidelines to only see patients with urgent medical needs. As a result, some AYA survivors expressed frustration and anger when told their health concerns were deemed non-urgent and, thus, had their care delayed, including visits with their healthcare providers, labs, and scans. For example, an AYA survivor stated, "I don't care where on the totem pole you think I am, but I think I'm important enough to get that treatment." (Female, FG 2).

Additionally, due to the presence of COVID-19 safety protocols (e.g., temperature checks and social distancing) in healthcare clinics, some survivors expressed being comfortable and feeling safe visiting healthcare clinics. For others, however, COVID-19 safety protocols did little to reduce their anxiety and fear of contracting the virus at the clinic. Instead, safety precautions caused some survivors to experience increased anxiety and concern that they may have symptoms of COVID-19. Due to the timing of scheduled doctor appointments in relation to the pandemic and healthcare clinics' varying guidelines, some individuals reported only difficulties in accessing medications and other healthcare-related items such as hearing aids, but in general "nothing's changed" (Female, FG 6) concerning their cancer treatment and other medical care plans. For example, one AYA survivor stated, "I think I'm at the point where I go in every other year and don't have to go in until next-next year." (Male, FG 1).

On the other hand, other AYAs reported changes including unwanted telemedicine in place of physical visits, extended time between visits with their physicians, postponement of treatment involving clinical trials, and delays in scans and laboratory work (e.g., MRI, blood draws). One AYA survivor stated that "I really wanted to get the scans done. So, it was a little hard to have it delayed, but it's just something I have to deal with." (Female, FG 2). Delays in clinical treatments led to feelings of helplessness, and delays in scans led to heightened fear of recurrence. Additionally, survivors also stated difficulty in scheduling appointments. Consequently, limited access to healthcare resources was a source of uncertainty. Although lockdowns loosened later in the pandemic and more resources became available than at the very start of the pandemic, an AYA survivor stated,

But it's not as easy as it was prior to COVID, right? Where you could call and say, "I need my MRI." "Okay, what date are you available?" They're asking me. Now I'm asking them, "What do you have available?" And let me throw my whole schedule around just so I can make this appointment. Because I know if I don't go to this appointment this week, I have to wait six weeks from now." (Female, FG 2) 
Although telemedicine was crucial in helping individuals connect with their healthcare providers with minimal risk, AYAs also described downfalls to using telemedicine, such as doctors needing to rely on their patients to perform physical self-examinations. An AYA survivor described, "It feels like a lot of the onus is on the patient. Instead of providing actual medical care, they're just kind of giving you whatever you think you have is kind of what it feels like." (Female, FG 5). Individuals needing to be their "doctor's hands" described feelings of increased responsibility, stress, uncertainty, and anxiety.

Similarly, some AYAs also expressed feelings of distrust with their healthcare providers. Due to potential conflicts of interest, some individuals believed their health and safety was placed secondary to their healthcare clinic's financial interests. For example, one AYA said,

I think that, at least what I've experienced at the hospitals, like, I know that they are stressed, and they are losing out on all this money. And so it seems kind of like they're scheduling things for people by default. And when you even bring up, like, is it safe, like, I'm worried about it, they're...they admit that it's not really safe and that you should probably just reschedule. But they're scheduling everybody even though it's not safe necessarily. And so that's really not reassuring. (Male, FG 5)

Individuals also expressed feeling reluctant to visit their healthcare provider despite being encouraged by their healthcare provider and "felt like they [healthcare providers] weren't taking it [COVID-19] as seriously as they should" (Female, FG 3). This may have been due to different risk perceptions between healthcare providers and patients.

Among a subset of AYA's who were in active treatment, particularly among those who had a recurrent disease or a terminal diagnosis, the disruption in care was incredibly traumatic. For those AYAs, clinical trials often represent the only available treatment options. As one AYA put it,

I've exhausted most of my baseline courses of treatment, so clinical trials are essential for me. And when you hear, like, oh, there's one where you are an ideal fit, you fit all of the inclusion criteria, there's no exclusion bumping you out, this looks like a perfect fit for you, but you can't do anything about it, because we're on hold and don't know when we're going to get off of hold. That's a little bit nerve-racking. (Male, FG 3)

Similarly, another AYA described her difficulties with clinical trials being placed on hold:
My last scan showed that my cancer progressed, and we were hoping to move me onto a trial. And yeah, they're on pause right now. So right now, the next course of treatment would be one of the drugs on the trial and we're hoping that we can then make an appeal to my insurance company for a compassionate whatever that they would let me use the other drug that is showing promising results in the trial. (Female, FG 3)

Mental health stressors AYAs also described mental health stressors during the early stages of the pandemic above and beyond those of their peers without cancer. Many participants expressed frustration due to people around them, including family members and friends, "not taking it seriously" when it comes to COVID-19 precautions. Some described arguments with loved ones about the need to wear masks and practice social distancing. Many other AYAs shared the frustration they felt when seeing social media posts with peers who were "not doing what they're supposed to be doing" (Female, FG 3). One survivor summarized this sentiment with,

And it's just incredibly frustrating to feel like a lot of people aren't taking it seriously. But for anything to actually change, and for us to move forward, everybody has to take it seriously. We've all been in the cancer boat, we know what it takes to adapt. Why can't these other fools do that as well? (Female, FG 2)

Off-treatment cancer survivors shared the feelings of anxiety around their perceived increased risk of COVID19 and the frustration that others do not understand this or assume they are overacting. For example, one survivor stated,

It's increased my anxiety quite a bit. It's made me really scared to go out in public... Because I don't look actively ill, so people don't think that I have any kind of issues with my health. So, I don't outwardly appear to have any kind of illness, which I think makes people think that I'm overreacting. (Female, FG 5)

Another AYA survivor suggested that friends and family have difficulty understanding their fears.

I think people who haven't been through cancer or who haven't been compromised, I think they just have trouble having empathy for those of us who have experienced that. And so, they can't see the severity of the situation. I've definitely seen that in a lot of my friends and family. (Female, FG 2) 
Compounding uncertainty Across all focus groups, AYAs described feelings of uncertainty as being particularly difficult during the early stages of the pandemic. In fact, when asked about their top challenges faced during the pandemic to date, uncertainty ranked at the top for most. AYAs described feelings of uncertainty related to the unknown end date of the COVID-19 pandemic and, subsequently, their inability to make plans for the future. Several AYAs compared and contrasted the pandemic to their initial cancer diagnosis and said that the lack of a "timeline" for the COVID-19 pandemic made it particularly challenging. For example, one survivor stated,

When I was in treatment, I knew I was going to have eight treatments. It was going to take six months. I knew I would be hopefully done at the end of that. But with this, they said two weeks, and here we are four months later. So, there is not really a solid end. And the feeling of not knowing when you're going to be able to... or if you're going to be able to return to anything that you used to do has been very unsettling and very hard. (Female, FG 5)

Another area of uncertainty for survivors was around their risk perceptions around COVID-19; many were not sure whether they were more susceptible than their peers to COVID or whether they may have more severe symptoms due to their history of cancer treatment. Some uncertainty stemmed from having been out of treatment for a long time and due to delayed surveillance appointments, not having an understanding from the doctor where they stood in terms of risk. One survivor shared,

We don't know if there is other health issues that are going to come up, like if history [of cancer] is going to somehow make a COVID case worse or it would make me more susceptible. (Male, FG 1)

Related to this risk uncertainty was the difficulty in weighing decisions about screening and other medical treatments. Participants described having to make decisions about whether certain screenings or tests were worth the risk of exposure in medical facilities. One AYA stated, "So every day is just kind of a, to me, it's kind of weighing my options. What's worse, getting a recurrence or getting COVID?" (Female, FG 3). These types of decisions were particularly difficult as AYAs struggled with trust with the healthcare industry's priorities (i.e., profits versus safety) as perceived by AYAs, described above.

\section{Unexpected advantages of a cancer history}

The early stages of the pandemic created disruptions in daily living for AYAs that were similar to those experienced by the general public. However, being a cancer survivor created unique medical care challenges, amplified existing, and created new anxieties and magnified uncertainties. Yet, the survivorship experience translated to advantages in coping and developing resilience.

Coping strategies In the wake of social distancing and dealing with the global pandemic, AYAs who were already dealing with depression and anxiety from their cancers now had the added burden of COVID-19. Figuring out ways to cope was paramount. One AYA survivor said:

I've been trying to talk to a lot of friends and stay in touch with people. Because I think for me one of the hardest parts is the isolation piece. And struggling with depression and wanting to isolate on my own, and then being forced to, is its own different thing. And it's really hard. And so, trying to fill my time, because I'm stuck at home all day, and just getting a schedule and trying to reach out to friends more often. (Female, FG 2)

Some AYAs found it helpful that they already had medication for anxiety and depression on hand for dealing with their cancer. One AYA explained that having medication already meant "that has definitely smoothed the path for me a little bit through COVID." In some ways, cancer had also taught them how to self-isolate. While it was nothing new, it was still challenging to do. Some AYAs turned to methods they had relied on during the cancer treatments. One survivor mentioned:

I feel like I kind of reverted back to my old coping mechanisms for when I was immunocompromised, and I had to stay home. I literally still have all the old coloring books and everything that I used to have from then, and I was just doing them again because I didn't know what else to do. And I was like, well, it worked then, so it might work now. (Female, FG 2)

Again, AYAs relied on strategies they previously used to cope. For many, exercising was a good outlet for dealing with anxiety and depression added by the pandemic. Others walked a lot more, although in some cases, walking also meant finding ways to avoid crowds or going to remote locations. For a majority of AYAs, they turned to online support platforms (e.g., The Cancer Patient, Elephants \& Tea) they used during their cancer journey. 
Resiliency AYAs perceived that their cancer had conferred some measure of resilience that helped them deal with the many disruptions created by the pandemic. For AYAs who felt like they already survived worse with their cancer, social distancing due to COVID-19 had "really not been that bad." While COVID-19 brought many challenges, many AYAs expressed familiarity with quarantining and feeling like they had a leg up on the general public after dealing with their own cancer journeys that prepared them to live with uncertainty and to be hyperaware of their health and doing things that could affect their immune-compromised selves. While cancer was not a positive experience, quarantining was something they already knew how to do and were able to transition because they had "already been through it and know what to expect." Some AYAs also noted that the pandemic created a more level playing field for others to empathize with cancer survivors and to understand what sickness uncertainty felt like. One AYA survivor said:

But for the first time I feel like other people who haven't had cancer realize what the fear of getting sick is. Because I was scared about getting sick before this happened, just, it wasn't of COVID, it was of relapse. Well, now people who don't have COVID are scared they're going to get COVID. And I feel like, for the first time, people are on my level. (Female, FG 3)

Furthermore, cancer was seen as providing AYA survivors a head-start to deal with the pandemic. For example,

I feel like I have an advantage to some aspect on the mental game we're playing. Because I know what it's like. So I noticed, really when this all started, where people were like, 'Oh, my gosh, we need masks. Oh my gosh, all this.' Like, you guys are all freaking out. I've been doing this for four years. Like, I'm going to put a mask on and go to the store. Like, chill out. It's not the end of the world. So, I feel like people are starting to understand what it's like to not have a certain future. And everybody's response to that is, to some extent, funny to me, because you guys all gave me crap about it for the last four years. And now you're starting to be like, 'Oh, wow, this is legit. Like, it's scary.' And it's like, 'Yeah, it is.' Shocker. (Female, FG 3)

\section{AYA recommendations and resources}

When AYAs were asked what resources they would find most helpful for cancer patients and survivors during the pandemic, one AYA survivor recognized that friends and family could not help with issues of unemployment or healthcare access but that they could offer social support. "I think for me, it's just a simple phone call [from loved ones], simple phone calls go through a long way with me" (Male, FG 1). The sentiment of having support from friends and family was commonly echoed by the majority of AYA participants.

Besides support from family and friends, AYAs also stated that they wanted greater assistance in finding a young adult cancer community, an increased focus on mental health for their survivorship plan, and protection of wellness services during the pandemic. During the pandemic, support care and mental support were deemed nonessential, and as a result, survivors were left scrambling for resources. For example, one AYA survivor said:

I don't know if it's necessarily just pandemic wise, but at least for me and others I've talked with, young adults really aren't told about other young adult people or groups or things to do outside of getting sick. Like, I didn't know anybody, and it was all on me to figure it out. But I walk in the hospital and there's flyers for all, like, breast cancer groups and, you know, older adult groups. And it seems like the young adult community is something that isn't talked about, isn't looked at." (Female, FG 3)

Some AYAs expressed needing more financial assistance from the government (e.g., more than one stimulus check) and/or assistance from a nonprofit organization for groceries and other necessities. One AYA survivor requested more transparency and information surrounding the pandemic so that, "people can truly understand what's going on and how different things that they do might affect the world" (Male, FG 1). In terms of research, one AYA in active cancer treatment requested more studies and statistics on COVID-19 specifically related to cancer patients and survivors.

\section{Discussion}

In general, the early stages of the COVID-19 pandemic impacted both AYAs' daily living and cancer care. AYA survivors in our study tended to follow COVID-19 safety guidelines (e.g., wearing a mask and social distancing). As a result, survivors attempted to limit their "outdoor" activities and were frustrated at other individuals for not taking corresponding precautions against COVID-19. Negative impacts included reduced incomes, lost job opportunities, being the "bad guy" in the family, feelings of isolation, and struggles with depression. These factors likely contributed to the finding that the majority of AYA felt more isolated due to the pandemic. This isolation seemed to cross over both feelings in the personal life of AYA as well as the changes in their access to and navigation of medical care. Positive impacts included having more 
time to reconnect with family and friends, gaining support from family and friends, and discovering new support resources.

The impact of the COVID-19 pandemic on AYA's cancer treatment varied depending on which stage AYAs were on the continuum of care. For AYA survivors who planned to see their healthcare provider infrequently (e.g., once a year), the COVID-19 pandemic has had minimal impact on their treatment plans. However, AYAs who were undergoing treatment or on a more frequent surveillance schedule had a more difficult time accessing and navigating their medical care since appointments were being canceled and laboratory work and scans postponed. Most strikingly, because many oncology studies were postponed or temporarily suspended at the start of the pandemic [13], AYAs who relied on clinical trials for their medical care experienced the most disruptions in their treatment plans. For AYAs who had exhausted all other treatment options, the suspension of clinical trials left some AYAs with no alternatives but to wait in fear of recurrence or further progression. AYAs not enrolled on a clinical trial still experienced delays in care, mostly related to delayed or deferred clinical appointments. Dealing with cancer uncertainty and anxiety are well-documented issues among cancer patients and survivors. While previous studies regarding AYAs highlighted sources of uncertainty [14], the impact of illness uncertainty $[15,16]$, and coping strategies used by AYAs during times of uncertainty [17], they were not designed to understand the impact of compounding uncertainty caused by the COVID-19 pandemic and their cancer treatment. However, one recent survey study focused on the most common effects of the early stages of COVID-19 on AYAs related to uncertainty [18]. Specifically, the survey found that AYAs most often reported, "not being sure how big of a risk COVID-19 posed for them or how their body would react to the infection" and "being anxious about experiencing serious complications if they were to catch the virus." Similarly, in our focus groups, most AYAs ranked uncertainty as their primary concern. Additionally, our results provided further insight in that AYAs discussed the multiple layers of uncertainty and associated worry. AYAs still faced the typical cancer uncertainties (e.g., fear of recurrence, late effects symptoms, unease about physical symptoms and what they might mean) and now have added concerns about the unknowns of their possible increased risk and morbidity from COVID-19.

Related to these compounding layers of uncertainty, AYAs discussed their difficulty in making risk assessments in determining how to respond to COVID-19. Risk perceptions varied based on current treatment status, length of time since completion of treatment, and having other comorbid health conditions. AYAs struggled to understand this risk and how the varying guidelines in response to the pandemic (e.g., quarantining) would impact or change risk. While unknown risk is not unique to AYA cancer patients and survivors [19,
20], the unclear risk perception among AYAs created additional stress around medical decision-making. At times, for example, it was not clear to AYAs whether it was safer to postpone treatment or surveillance care and risk possible recurrence or to attend scheduled appointments when they were available and risk potential COVID-19 exposure. Uncertainty about risk suggests that healthcare practitioners may need to help AYAs assess risk, which could influence day-to-day actions. Additionally, AYAs likely need increased psychosocial supports to manage the anxiety from compounding uncertainty and unclear personal risk. Many AYAs reported that clear public health guidance and policies, such as mandatory masking and limited occupancy guidelines, eased the effects of uncertainty because it provided boundaries for both themselves and their friends and families that allowed them to make choices about daily living with less anxiety.

In response to the pandemic, many healthcare facilities added telehealth or virtual visits to bridge healthcare needs. This approach worked well for some AYAs and was even favored by some who said that without the busy clinic schedules of the past, they felt like virtual visits gave them more time with their provider to ask questions and discuss concerns. However, for other AYAs, virtual visits created anxiety, particularly for visits and procedures that would normally require a healthcare provider's touch (i.e., feeling the thyroid to check for lumps). This is a key area medical systems need to address for some patients, as it became an added burden resulting in anxiety and uncertainly about that nature, quality, and possible outcomes related to care.

An unexpected silver lining noted by AYAs was that having had cancer conferred some advantages for coping. Knowing how to handle isolation, having medications on hand for depression and anxiety, and having completed cancer treatment translated to feelings of resilience for dealing with a global pandemic. The ability to recall and use past strategies for success is one that can be helpful in serving AYAs to cope with current stressors and uncertainty in the short term.

While our study helped to examine the impact of COVID19 on AYAs and provides new information about this understudied population, there are some limitations. First, our sample was predominantly White and included only seven non-White participants and notably no African American AYAs. Therefore, the particular challenges and impact of COVID-19, including greater prevalence and death among minority (particularly African American) communities, are not documented here. Although we sent recruitment materials to African American and Latino networks, this effort yielded little. It is important that the experiences of communities of color are reflected in the COVID-19 impact stories so that needs are documented to best be able to find solutions that are culturally appropriate and needed. Additionally, our sample included AYAs aged 18-39, and thus the results may not be relevant to younger AYAs (aged 15-17 years). Second, our 
survey instrument regarding impacts of COVID-19 was limited; particularly the item that was worded as "anxiety due to worry about my health or my family's health," which was endorsed by $90 \%$ of our sample. Due to the item's imprecision, it is impossible to determine whether this anxiety was regarding their own health or those of their family members or both. In their qualitative responses, AYAs discussed both types of anxiety, but future studies should consider expanding this to two separate survey items. Finally, the nature and response to the pandemic have been changing. Our study captured a snapshot in time to help us discern overarching issues that could be in the service of improving care for AYAs. Now that vaccines for COVID-19 are available, AYAs might assess their risk differently, which in turn may impact feelings of uncertainty. Therefore, it is important to assess changes throughout a crisis to ascertain differences in need and responses required. Considering that perspectives and outlooks may change throughout the COVID-19 pandemic, it is important to note that Table 2 represents AYA responses during the first three to four months (March-June 2020) of the pandemic.

\section{Practice implications}

Our findings pinpoint areas for healthcare providers and healthcare systems to consider in assuring the continuity of care for AYAs and ways for AYAs to best help manage their care. First, healthcare providers should triage who should receive virtual vs. in-person care and engage in active dialogue with patients about preferences when possible. Outside of those in active treatments, AYA survivors reported high levels of discomfort and anxiety at having to feel for nodes or selfassess whether there were certain changes in parts of their bodies. While some virtual visits served to stem the disruption of care during the pandemic, for other AYAs, utilizing phone or video visits was not ideal and served only to exacerbate feelings of anxiety. Second, AYAs did not understand to what degree their risk was heightened for COVID-19 due to having had a history of cancer and to what degree the time from active treatment played a role. Additionally, they had no central place for receiving information regarding risk. As the pandemic unfolded and guidelines rapidly changed, what these changes meant for how AYAs should operate on a day-to-day basis was not always clear as AYAs still wrestled with understanding COVID-19 and their risk. As knowledge about COVID19 advances, it is important for healthcare providers to be more explicit about risks, meanings, and how to manage. AYAs suggested there be a central repository for this kind of trustworthy information. This might include consensus updates posted through trusted agencies such as the NCI (National Cancer Institute) or guideline-based sources such as NCCN (National Comprehensive Cancer Network) or ASCO (American Society of Clinical Oncology) or more patient-facing sites such as the CDC (Centers for Disease Control) under a highlighted population tab. Finally, healthcare providers can focus on highlighting resources for psychosocial support for AYAs. In particular, AYAs benefitted from peer support networks (e.g., Elephants and Tea; The Cancer Patient) and online peer support groups. AYAs suggested that healthcare providers offer/suggest these resources where they may be highly visible, such as on practice websites.

For AYAs, the pandemic called out the need for psychosocial supports, especially around managing anxiety and uncertainty. Even though the pandemic brought along many stressors, AYAs overwhelmingly discussed that their cancer experience had provided a foundation for coping that they could leverage to navigate the challenges of the pandemic. While their cancer experience was not touted as a positive life change, they saw the value in having survived and could draw on those lessons to move forward. It is important that in times of crisis, AYAs are encouraged to examine what coping skills were meaningful during their cancer experience and evaluate whether they are valuable for the current issue. Public health experts expect another crisis or pandemic that we are likely to face in the future. As such, the lessons and experiences offered by the AYAs can serve to prepare us to manage future crises.

Supplementary Information The online version contains supplementary material available at https://doi.org/10.1007/s11764-021-01069-9.

Acknowledgements We would like to thank Chiu Feng Yap for her help in coding the focus group data. Additionally, we are thankful to Nicholas Giallourakis, Executive Director, Steven G. Cancer Foundation, and Angelike Giallourakis, PhD, President, Board of Directors, Steven G. Cancer Foundation, for their careful review of the manuscript and their support in recruitment through Elephants and Tea. Finally, we would like to thank Allison Grimes, MD, for her careful review and thoughtful comments on the manuscript.

Availability of data and material Not applicable

Code availability Not applicable

Author contribution All authors (LAS, MA, AL) made substantial contributions to the conception or design of the work; or the acquisition, analysis, or interpretation of data; or the creation of new software used in the work; drafted the work or revised it critically for important intellectual content; approved the version to be published; and agree to be accountable for all aspects of the work in ensuring that questions related to the accuracy or integrity of any part of the work are appropriately investigated and resolved.

\section{Declarations}

Ethics approval All study procedures were approved by the institutional review board at the University of Texas Health Science Center in Houston (HSC-SPH-20-0463).

Consent to participate Informed consent was obtained from all individual participants included in the study. 
Consent for publication Not applicable

Conflict of interest The authors declare no competing interests.

\section{References}

1. Centers for Disease Control and Prevention. About COVID-19. https://www.cdc.gov/coronavirus/2019-ncov/cdcresponse/aboutCOVID-19.html Accessed February 3, 2021.

2. World Health Organization. Coronavirus disease (COVID-19) pandemic. https://www.euro.who.int/en/health-topics/healthemergencies/coronavirus-covid-19/novel-coronavirus-2019-ncov Accessed February 3, 2021.

3. Centers for Disease Control and Prevention. Symptoms of Coronavirus. https://www.cdc.gov/coronavirus/2019-ncov/ symptoms-testing/symptoms.html Accessed February 3, 2021.

4. Kuderer NM, Choueiri TK, Shah DP, Shyr Y, Rubinstein SM, Rivera DR, et al. Clinical impact of COVID-19 on patients with cancer (CCC19): a cohort study. Lancet (London, England). 2020. https://doi.org/10.1016/s0140-6736(20)31187-9.

5. Wang H, Zhang L. Risk of COVID-19 for patients with cancer. Lancet Oncol. 2020;21:e181. https://doi.org/10.1016/s14702045(20)30149-2.

6. Marjerrison S, Barr RD. Unmet survivorship care needs of adolescent and young adult cancer survivors. JAMA Netw Open. 2018;1: e180350. https://doi.org/10.1001/jamanetworkopen.2018.0350.

7. Barr RD, Ferrari A, Ries L, Whelan J, Bleyer WA. Cancer in adolescents and young adults: a narrative review of the current status and a view of the future. JAMA Pediatr. 2016;170:495-501. https:// doi.org/10.1001/jamapediatrics.2015.4689.

8. Ueda M, Martins R, Hendrie PC, McDonnell T, Crews JR, Wong TL, et al. Managing cancer care during the COVID-19 pandemic: agility and collaboration toward a common goal. J Natl Compr Cancer Netw. 2020;18:366-9. https://doi.org/10.6004/jnccn.2020. 7560.

9. Jetelina KK, Knell G, Molsberry RJ. Changes in intimate partner violence during the early stages of the COVID-19 pandemic in the USA. Inj Prev. 2020;27:93-7. https://doi.org/10.1136/injuryprev2020-043831.

10. Knell G, Robertson MC, Dooley EE, Burford K, Mendez KS. Health behavior changes during COVID-19 pandemic and subsequent "stay-at-home" orders. Int J Environ Res Public Health. 2020;17. https://doi.org/10.3390/ijerph17176268.

11. Forman J, Damschroder L. Qualitative content analysis. In: Jacoby L \& Siminoff LA, editors. Empirical research for bioethics: A primer. 2008:39-62.

12. Glaser BG. The constant comparative method of qualitative analysis. Soc Probl. 2014;12:436-45. https://doi.org/10.2307/798843.

13. Ledford H. Coronavirus shuts down trials of drugs for multiple other diseases. Nature. 2020;580:15-6. https://doi.org/10.1038/ d41586-020-00889-6.

14. Donovan EE, Brown LE, LeFebvre L, Tardif S, Love B. The uncertainty is what is driving me crazy the tripartite model of uncertainty in the adolescent and young adult cancer context. Health Commun. 2014;30:702-13. https://doi.org/10.1080/10410236. 2014.898193.

15. Panjwani AA, Marín-Chollom AM, Pervil IZ, Erblich J, Rubin LR, Schuster MW, et al. Illness uncertainties tied to developmental tasks among young adult survivors of hematologic cancers. J Adolesc Young Adult Oncol. 2019;8:149-56. https://doi.org/10.1089/ jayao.2018.0024.

16. Corbeil A, Laizner AM, Hunter P, Hutchison N. The experience of uncertainty in young adults with cancer. Cancer Nurs. 2009;32: E17-27. https://doi.org/10.1097/ncc.0b013e3181a5690d.

17. Lie NEK, Larsen TMB, Hauken MA. Coping with changes and uncertainty: a qualitative study of young adult cancer patients' challenges and coping strategies during treatment. Eur J Cancer Care (Engl). 2018;27:e12743. https://doi.org/10.1111/ecc.12743.

18. Košir U, Loades M, Wild J, Wiedemann M, Krajnc A, Roškar S, et al. The impact of COVID-19 on the cancer care of adolescents and young adults and their well-being: results from an online survey conducted in the early stages of the pandemic. Cancer. 2020;126: 4414-22. https://doi.org/10.1002/cncr.33098.

19. Lanciano T, Graziano G, Curci A, Costadura S, Monaco A. Risk perceptions and psychological effects during the Italian COVID-19 emergency. Front Psychol. 2020;11. https://doi.org/10.3389/ Ffpsyg.2020.580053.

20. Huang Y, Yang C. A metacognitive approach to reconsidering risk perceptions and uncertainty: understand information seeking during COVID-19. Sci Commun. 2020;42:616-42. https://doi.org/10. 1172/F1075547020959818.

Publisher's note Springer Nature remains neutral with regard to jurisdictional claims in published maps and institutional affiliations. 